\title{
ORIGINAL ARTICLE Proteomic analysis of muscle between hybrid abalone and parental lines Haliotis gigantea Reeve and Haliotis discus hannai Ino
}

\author{
$\mathrm{G} \mathrm{Di}^{1,2}, \mathrm{X} \mathrm{Luo}^{1}, \mathrm{~W} \mathrm{You}^{1}, \mathrm{~J} \mathrm{Zhao}^{1}, \mathrm{X} \mathrm{Kong}{ }^{2}$ and $\mathrm{C} \mathrm{Ke}{ }^{1}$
}

To understand the potential molecular mechanism of heterosis, protein expression patterns were compared from hybrids of Haliotis gigantea (G) and Haliotis discus hannai (D) using two-dimensional gel electrophoresis (2-DE) and matrix-assisted laser desorption/ionization time-of-flight/time-of-flight analyses. Expression differences were observed in muscle samples from the four groups with $673 \pm 21.0$ stained spots for $H$. discus hannai $q \times H$. discus hannai ơ (DD), $692 \pm 25.6$ for $H$. gigantea $q \times H$. gigantea ô $(\mathrm{GG}), 679 \pm 16.2$ for $H$. discus hannai $q \times H$. gigantea o $(D G)\left(F_{1}\right.$ hybrid) and $700 \pm 19$ for $H$. gigantea $q \times H$. discus hannai o $(G D)\left(F_{1}\right.$ hybrid). Different 2-DE image muscle protein spots had a mirrored relationship between purebreds and the $F_{1}$ hybrid, suggesting that all stained spots in $F_{1}$ hybrid muscle were on 2-DEs from parents. DD and DG clustered together first, and then clustered with GD, whereas the distance of DD and GG was maximal according to hierarchical cluster analysis. We identified 136 differentially expressed protein spots involved in major biological processes, including energy metabolism and stress response. Most energy metabolism proteins were additive, and stress-induced proteins displayed additivity or overdominance. In these 136 identified protein spots, hybrid offspring with additivity or over-dominance accounted for $68.38 \%$. Data show that a proteomic approach can provide functional prediction of abalone interspecific hybridization.

Heredity (2015) 114, 564-574 doi:10.1038/hdy.2014.124; published online 11 February 2015

\section{INTRODUCTION}

The abalone is an aquatic animal of economic importance and China produces the most abalone (exceeding 50000 tons per annum). In China, long-term artificial reproduction and rafferty mating of farmed abalone has led to severe inbreeding and germplasm degradation manifested by individual miniaturization and poor disease resistance. Along with deterioration of the aquacultural environment, disease outbreaks and other issues cause massive deaths of cultured abalone (Zhang et al., 2004). Recently, hybridization has been shown to be effective and useful for shellfish genetic improvement programs (Elliott, 2000). Heterosis, or the strengthening of different hybrid characteristics, suggests the possibility of obtaining genetically superior individuals by combining parental virtues.

In China, Haliotis discus hannai Ino is an economically important gastropod with large-scale cultivation dating back to the late 1980s (Luo et al., 2010). Several problems emerged in the $H$. discus hannai industry associated with long-term cultivation, such as quality degeneration, and declines in disease resistance. Haliotis gigantea is a valued commercial species along the coast of Japan, and our research team first introduced $H$. gigantea from Japan into the Fujian Province for mariculture in 2003 and subsequent artificial propagation was successful (Luo et al., 2010). The meat of $H$. gigantea is crisp and tender, and $H$. gigantea has excellent disease resistance (Luo et al., 2010), all traits that make it an important commercial aquaculture species for China. To improve growth and disease resistance, hybridization of $H$. gigantea and $H$. discus hannai was performed. With karyotype analysis, amplified fragment length polymorphism, microsatellite and mitochondrial $16 \mathrm{~S}$ rRNA and cytochrome $c$ oxidase subunit I gene sequences, morphological, cytological and molecular characterizations of hybrids and pure progenies were conducted, and hybrid status was confirmed (Luo et al., 2010). We confirmed (in 2012) that the growth rate of hybrids was faster than $H$. discus hannai and $H$. gigantea at 100 days postfertilization. Survival of the reciprocal hybrid was significantly higher than $H$. discus hannai as well. Previous work (Luo 2009; Luo et al., 2013) indicated that interspecific hybrid abalone ( $H$. discus hannai $\times H$. gigantea) grow better and have greater environmental adaptability than parental species at 540 days postfertilization; thus the hybrid exhibits heterosis.

Although hybrid vigor has been exploited in agricultural crops, the molecular mechanisms responsible for this basic biological phenomenon are not well understood (Xiang et al., 2013). Recently, some studies suggest that hybridization between two parents can cause changes in gene expression, which may be responsible for heterosis (Xiang et al., 2013). Although relatively new genomics for genetic breeding studies concentrate on transcriptomics, the value of proteomic analysis is appreciated (Karr, 2007). Complex regulatory routes from posttranslational modifications to protein turnover cannot be studied at the cDNA level, and changes in gene expression

${ }^{1}$ State Key Laboratory of Marine Environmental Science, College of Ocean and Earth Sciences, Xiamen University, Xiamen, PR China and ${ }^{2}$ College of Fisheries, Henan Normal University, Xinxiang, PR China

Correspondence: Professor C Ke, State Key Laboratory of Marine Environmental Science, College of Ocean and Earth Sciences, Xiamen University, Xiamen, Fujian Province 361005, PR China.

E-mail: chke@xmu.edu.cn

Received 8 June 2014; revised 12 November 2014; accepted 14 November 2014; published online 11 February 2015 
do not necessarily reflect the changes in protein abundance (Xiang et al., 2013). Although transcriptomic analysis has increased our understanding of the heterosis, changes at the mRNA level may not indicate changes at the protein level (Guo et al., 2013).

Proteins are effectors of genetic information and have specific biological functions. Proteins, as performers of gene function, determine phenotypes, which can be thought of as snapshots of genome expression (Jin et al., 2014). Individual protein expression can be treated as inheritance of quantitative characteristics, even without prior information of protein identity, as most often relates to non-model species (Vasemägi and Primmer, 2005). Lately, twodimensional gel electrophoresis (2-DE) has been used to determine correlations between polymorphisms of individual proteins and hybrid vigor for agronomic traits (Xie et al., 2006; Hoecker et al., 2008; Dahal et al., 2012). Proteomic approaches can help characterize molecular phenotypes of hybrids compared with parental species and may identify relevant genetic markers (Guo et al., 2013). Thus proteomics holds promise for sustainable agriculture (Eldakak et al., 2013).

No hypothesis adequately explains heterosis, so further exploration is needed. Several studies suggest that proteomic application between maize hybrids and their parents can identify posttranscriptional protein differences (Hoecker et al., 2008; Muthreich et al., 2010; Fu et al., 2011), and proteins related to heterosis during maize germination stages have been identified (Hoecker et al., 2008). The observed patterns indicate important roles of dominance, partial dominance and over-dominance in maize (Fu et al., 2011). Also, dominance could be the major molecular basis of wheat root heterosis (Thiemann et al., 2009).

Proteomics has been used in plants, but marine invertebrate use is limited. Ours represents the study in abalone to study differential protein expression between hybrid and parents to elucidate molecular mechanisms behind heterosis (Xiang et al., 2013). Using 2-DE and mass spectrometry (MS), we compared the muscles from hybrid and parent abalone to dissect genetic mechanism of heterosis. We measured differences in muscle protein abundance in two species and studied proteomic expression changes within and between four hybrid abalone groups.

\section{MATERIALS AND METHODS}

\section{Animal materials}

$H$. gigantea $\$ \times H$. gigantea ơ $(\mathrm{GG}), H$. discus hannai $\times H$. discus hannai o (DD), $H$. discus hannai $\uparrow \times H$. gigantea ${ }^{+}(\mathrm{DG})$ and $H$. gigantea $\uparrow \times H$. discus hannai ơ (GD) were produced at JieFeng Abalone Farm in Fuzhou City, China. Samples of hybrid and pure bred offspring of $H$. gigantea and $H$. discus hannai were obtained in 2006. The four abalone groups (GG, DD, DG and GD) were strictly isolated in the farming process, and culture conditions were the same. Random sampling took place from four cultured populations, and the four abalone family sizes are not significantly different. External features of the four abalone families can be found in Supplementary Figures S1 and S2). For each group (DD, DG, GD or GG), muscle proteins were extracted from each of the 18 abalones in three independent samples, each of which contained the muscles from six abalones; three technical replicates were performed for each sample to ensure reproducibility.

\section{Extraction of abalone muscle proteins}

The abalone foot was used and this has been recommended by previous research because of ease of isolation and less vulnerability to parasitic infection (Diz and Skibinski, 2007). Sample preparation was identical to that depicted in the literature (Di et al., 2013). Muscle samples were collected from a central incision in the pedal muscle. Briefly, a sample of $20 \mathrm{mg}$ was crushed in liquid nitrogen, and the resultant powder was suspended immediately in $1 \mathrm{ml}$ Trizol. Protein extraction was performed as previously described with Trizol-mediated sample preparation (Di et al., 2013). The dry protein pellet was re-suspended in isoelectric focusing re-dissolving buffer $(7 \mathrm{M}$ urea, $2 \mathrm{M}$ thiourea, $4 \%(\mathrm{w} / \mathrm{v})$ CHAPS, $40 \mathrm{mM}$ Tris). Protein concentration was measured with a Protein 2-D Quant Kit (GE Healthcare, Piscataway, NJ, USA).

Twenty-microgram aliquots of the muscle proteins extracted from each of the 18 abalones were used. The 18 aliquots were combined into three subgroups each containing six samples. Each subgroup contained $120 \mu \mathrm{g}$ protein, representing a biologically duplicate set, and all sets were stored at $-70{ }^{\circ} \mathrm{C}$ prior to the experiment.

\section{Two-dimensional gel electrophoresis}

First, $120 \mu \mathrm{g}$ protein $(340 \mu \mathrm{l}$ solution) was loaded onto a $18-\mathrm{cm}$ IPG strip (Amersham Biosciences, Piscataway, NJ, USA) with $\mathrm{pH} 4-7$ (linear) to allow for a 13-h active rehydration, followed by isoelectric focusing in an electrophoresis apparatus (Bio-Rad, Laboratories, Hercules, CA, USA) at $20^{\circ} \mathrm{C}$. Electrophoresis was run at $100 \mathrm{~V}$ for $2 \mathrm{~h}$, followed by a linear increase from 100 to $8000 \mathrm{~V}$ and at $8000 \mathrm{~V}$ for a total of $50000 \mathrm{Vh}$. The current did not exceed $50 \mathrm{~mA}$ per strip. Before the second dimension, focused IPG strips were equilibrated for $17 \mathrm{~min}$ in equilibration buffer I $(6 \mathrm{~m}$ urea, $50 \mathrm{~mm}$ Tris- $\mathrm{HCl}$ buffer ( $\mathrm{pH} 8.8), 2 \%$ sodium dodecyl sulfate, $30 \%$ glycerol, and $1 \% \mathrm{w} / \mathrm{v}$ dithiothreitol) followed by $17 \mathrm{~min}$ in equilibration buffer II (same as buffer I, but dithiothreitol replaced with $2.5 \%$ iodoacetamide). The second dimension of gel electrophoresis was carried out on $12.5 \%$ polyacrylamide gels using protean Xi Cell (Bio-Rad) and sealed with $0.5 \%$ w/v agarose. Running buffer system was the standard buffer for sodium dodecyl sulfate-polyacrylamide gel electrophoresis. Gels were run for $30 \mathrm{~min}$ at $12.5 \mathrm{~mA}$ per gel and then at $25 \mathrm{~mA}$ per gel for about $6 \mathrm{~h}$ until the bromophenol blue marker reached the bottom of the gel. Gels were stained with silver and analyzed with MS (Di et al., 2013).

\section{Image acquisition and analysis}

For each abalone group, three independent samples, each of which comprised of muscle from six abalone, were used in 2-DE analysis, and three technical replicates were conducted for each sample to ensure reproducibility. The 2-D gels were scanned at an optical resolution of 300 dpi using an Image Scanner (Amersham Biosciences, UTA-1100). Qualitative and quantitative differences in spot intensity were analyzed with the PDQuest software (ver. 8.0; Bio-Rad). Software was used for spot detection, matching between gels and volume measurement. Automatic spot detection in each gel was verified by visual inspection to ensure that spots were all detected. Intensity detected during image acquisition was expressed as the relative volume of the spots in each gel. Only well-resolved spots were used, discarding overlapping and streaked areas or spots near the edges.

\section{Statistical analysis}

Data analysis of 'spot' and 'genotype'. Statistical data analysis was conducted using the SPSS version 13.0 software. Skewing in spot volume was removed by $\log _{10}$ transformation spot volume according to a previous recommendation (Karp et al., 2005). Only dots presented in all technical and sample replicates were included in analysis (Diz and Skibinski, 2007). To compare the four groups together, analysis of variance was used.

Hierarchical clustering and genetic distances of the four abalone groups. Hierarchical clustering with a TMEV heatmap showing all treatments and normalized spot volumes for each spots across the gels was constructed (Saeed et al., 2006). Genetic distances between lines were calculated from each protein fraction, allowing two distinct genetic groups of four lines to be distinguished.

Data analysis of differentially expressed proteins identified. Intensities for all protein spots for the four abalone groups were generated as percentage values, which were then compared for significant differences between the four groups. Spot analysis was performed using both qualitative and quantitative modes using the Student's $t$-test. For quantitative analysis, the $t$-test was conducted for many spot pairs, and spots with at least 1.5 -fold changes among the four groups were compared and considered upregulated or downregulated. Protein spots detected quantitatively were conducted between 'any two groups'. For qualitative analysis, spots with at least 10 -fold changes were considered absent/present. 


\section{In-gel digestion of proteins}

Spots differentially expressed were excised for MS identification. Briefly, protein spots were washed for $10 \mathrm{~min}$ with water, destained using $15 \mathrm{~mm}$ potassium ferricyanide and $50 \mathrm{~mm}$ sodium thiosulfate and washed three times with Milli-Q $\mathrm{H}_{2} \mathrm{O}$. Spots were then shaken in $200 \mu \mathrm{l} 200 \mathrm{~mm}$ ammonium bicarbonate $\left(\mathrm{NH}_{4} \mathrm{HCO}_{3}\right)$ solution for $20 \mathrm{~min}$ and dehydrated using $200 \mu \mathrm{l}$ acetonitrile for $10 \mathrm{~min}$, and then the acetonitrile was removed. The gel pieces (protein spots) were dried, and proteins were reduced by treatment with $10 \mathrm{~mm}$ dithiothreitol for $30 \mathrm{~min}$ at $56^{\circ} \mathrm{C}$ and alkylated with $55 \mathrm{~mm}$ iodoacetamide. Trypsin digestion was performed by addition of $4 \mu$ of trypsin $\left(20 \mathrm{ng}^{-1}\right)$ in $20 \mathrm{~mm} \mathrm{NH}_{4} \mathrm{HCO}_{3}$ solution to each spot followed by incubation overnight at $37^{\circ} \mathrm{C}$.

\section{Matrix-assisted laser desorption/ionization time-of-flight/time-of- flight (MALDI-TOF-TOF) analysis}

For MALDI-TOF/TOF, peptides were extracted using $0.1 \%(\mathrm{v} / \mathrm{v})$ trifluoroacetic acid in $50 \%$ acetonitrile. Peptides were directly transferred onto a new target Typically, $0.8 \mu \mathrm{l}$ of peptides were mixed with $0.4 \mu \mathrm{l}$ of matrix solution $\left(2 \mu \mathrm{g} \mathrm{l}^{-1}\right.$ alpha-cyano-4-hydroxycinnamic acid in $0.1 \%$ trifluoroacetic acid and $50 \%$ acetonitrile), spotted onto MALDI target plates, followed by air drying and analyzed by a MALDI TOF/TOF MS.

MS analyses were conducted using an AB SCIEX MALDI TOF-TOF 5800 Analyzer (AB SCIEX, Xiamen, China) equipped with a neodymium: yttrium-aluminum-garnet laser (laser wavelength was $349 \mathrm{~nm}$ ), in reflection positive-ion mode. With alpha-cyano-4-hydroxycinnamic acid as the matrix and trifluoroacetic acid for an ionization auxiliary reagent, TOF/TOF calibration mixtures were used to calibrate the spectrum to amass tolerance within 150 p.p.m. MS spectra were processed using the TOF/TOF Series Explorer software (AB SCIEX) allowing non-redundant and fully automated selection of precursors for MS/MS acquisition. For the MS mode, peptide mass maps were acquired in positive reflection mode, and the $850-4000 \mathrm{~m} / \mathrm{z}$ mass range was used with 1000 laser shots per spectrum with a laser pulse rate of $400 \mathrm{~Hz}$, whereas in the MS/MS mode spectra up to 2000 laser shots were acquired and averaged with a pulse rate of $1000 \mathrm{~Hz}$. The PMF peak detection criteria used were a minimum signal-to-noise of 10 , a local noise window width mass/charge $(\mathrm{m} / \mathrm{z})$ of 250 and a minimum full-width at half-maximum (bins) of 2.9 A maximum of 20 most intense precursors per spot were selected for the MS/MS analysis, and peptides were fragmented with collision-induced decomposition with an energy of $1 \mathrm{kV}$. For collision-induced decomposition experiments, ambient air was used as collision gas with a medium pressure of $10^{-6}$ Torr (Wang et al., 2012).

The contaminant $\mathrm{m} / \mathrm{z}$ peaks originating from human keratin, trypsin autodigestion or matrix were included in the exclusion list used to generate the peptide mass list for the database search from the MS/MS analysis. For interpretation of the mass spectra, a combination of peptide mass fingerprints and peptide fragmentation patterns were used for protein identification in an NCBI non-redundant database (updated December 2010, containing 4607655 entries) (Wang et al., 2012). All database searches were fulfilled using the GPS Explorer software (version 3.6, AB SCIEX) running a Mascot search algorithm (v2.2, Matrix Science, London, UK) for protein identification. Protein identification was conducted using the multi-layered work-flow integrated mass spectra processing with conventional and homology-based searches. Briefly, Mascot, a highly specific stringent search, was applied as the firstlayer screen to identify either known proteins or unknown proteins sharing identical peptides presented in a database (Wang et al., 2012). Once the confident identifications were removed after searching against the NCBInr database, the rest were searched against the abalone-expressed sequence tag database.

\section{Database search criteria}

The raw MS and MS/MS spectra were processed with the following criteria: peak density filter, 50 peaks per $200 \mathrm{Da}$; maximum number of peaks, 65 ; and MS/MS peak filtering mass range, 60-200 Da. The searches were conducted using the following setting: taxonomy: all (NCBInr); enzyme: trypsin; monoisotopic/average: monoisotopic; missed cleavage sites allowed: 1; $P<0.05$ significance threshold; and carbamidomethylation of cysteine as fixed modification and methionine oxidation as variable modification; fragment mass tolerance: $0.25 \mathrm{Da}$; peptide mass tolerance: 50 p.p.m.; and MS/MS ion tolerance: $0.1 \mathrm{Da}$. Protein scores with confidence interval $(\mathrm{CI} \%)$ values $>95 \%$ were considered positively identified. The database result included the NCBI accession number, protein name, CI\% values, theoretical molecular weight and isoelectric points (pI). MS/MS spectra were subjected to similarity searches against the abalone EST database (downloaded from NCBI, updated December, $2010)$ using the BLASTX algorithm. The $E$-value was $<\mathrm{e}^{-20}$.

\section{Bioinformatic analysis}

The identified proteins were converted to their corresponding homologous GI numbers and imported into the searching web of a protein information resource (http://pir.georgetown.edu/pirwww/search/batch.shtml), and multiple entries were retrieved from the iProClass database. The protein sequence of those retrieved with GI numbers was then submitted to the Automatic Annotation Server ver. 1.64a of KEGG in FASTA format, and the identified proteins were then matched to specific processes or functions by searching Gene Ontology (www.geneontology.org) (Wang et al., 2012). The Gene Ontology project provides structured, controlled vocabularies and classifications that cover several domains of molecular and cellular biology and are freely available for community use in the annotation of genes, gene products and sequences.

\section{Subcellular location of the identified proteins}

Protein subcellular location is important to protein function. We can get FASTA sequences using the NCBI accession numbers from identified proteins and predictive methods (http://psort.hgc.jp/form2.html). Moreover, protein subcellular location can be predicted using the Proteome Analyst-Subcell Specialization (http://pa.wishartlab.com/pa/pa/index.html) with a procedure that consists of signing in, choosing a proteome processor and importing FASTA sequences.

\section{RESULTS}

\section{Analysis of 2-DE gels for muscle proteomes}

Protein spots observed on gels exceeded 700 per gel. Most spots were present on all gels, and others were present on all biological replicates of at least two of abalone samples analyzed. Silver staining had a linear sensitivity range of three orders of magnitude. Here the weakest and the strongest spots fell within this range. Representative examples of 2 -DE gels are shown in Figure 1. There were $673 \pm 21$ stained spots (three biological replicate gels, $n=3)$ for the DD, $692 \pm 25.6(n=3)$ for the GG, $679 \pm 16.2(n=3)$ for the DG and $700 \pm 19(n=3)$ for the GD groups. Obvious dominant spots were $\sim 85 \mathrm{kDa}$.

Differentially expressed protein spots were distributed among four abalone lines based on 2-DE gel image analysis. Different numbers of unique, paternally inherited, maternally inherited and relatively constant spots are depicted in Venn diagrams (Figure 2). We observed 600 spots shared by all four abalone lines and 169 differentially expressed spots. In addition, we found 20 and 24 spots shared among three possible schemes, DD/DG/GD and GG/DG/GD, respectively. There were 19, 24, 16 and 28 spots shared among four possible pairing schemes, DD/DG, DD/GD, GG/DG and GG/GD, respectively. GD shared more spots with GG than DD. There were 10 and 24 spots unique in DD and GG, respectively, compared with 4 unique spots in GD. There were 165 differently stained spots between DD and GG, and these were significantly greater than that between DD and DG $(n=74)$, between DD and GD $(n=85)$, between GG and DG $(n=91)$, between GG and GD $(n=88)$ and between DG and GD $(n=91)$. Differences between parent muscle proteins were higher than that between parent and filial generations. Moreover, differences between purebreds and offspring were close (DD versus DG and GG versus GD, DD versus GD and GG versus DG). Differences between F1 hybrids and male abalone muscle proteins were higher than between 

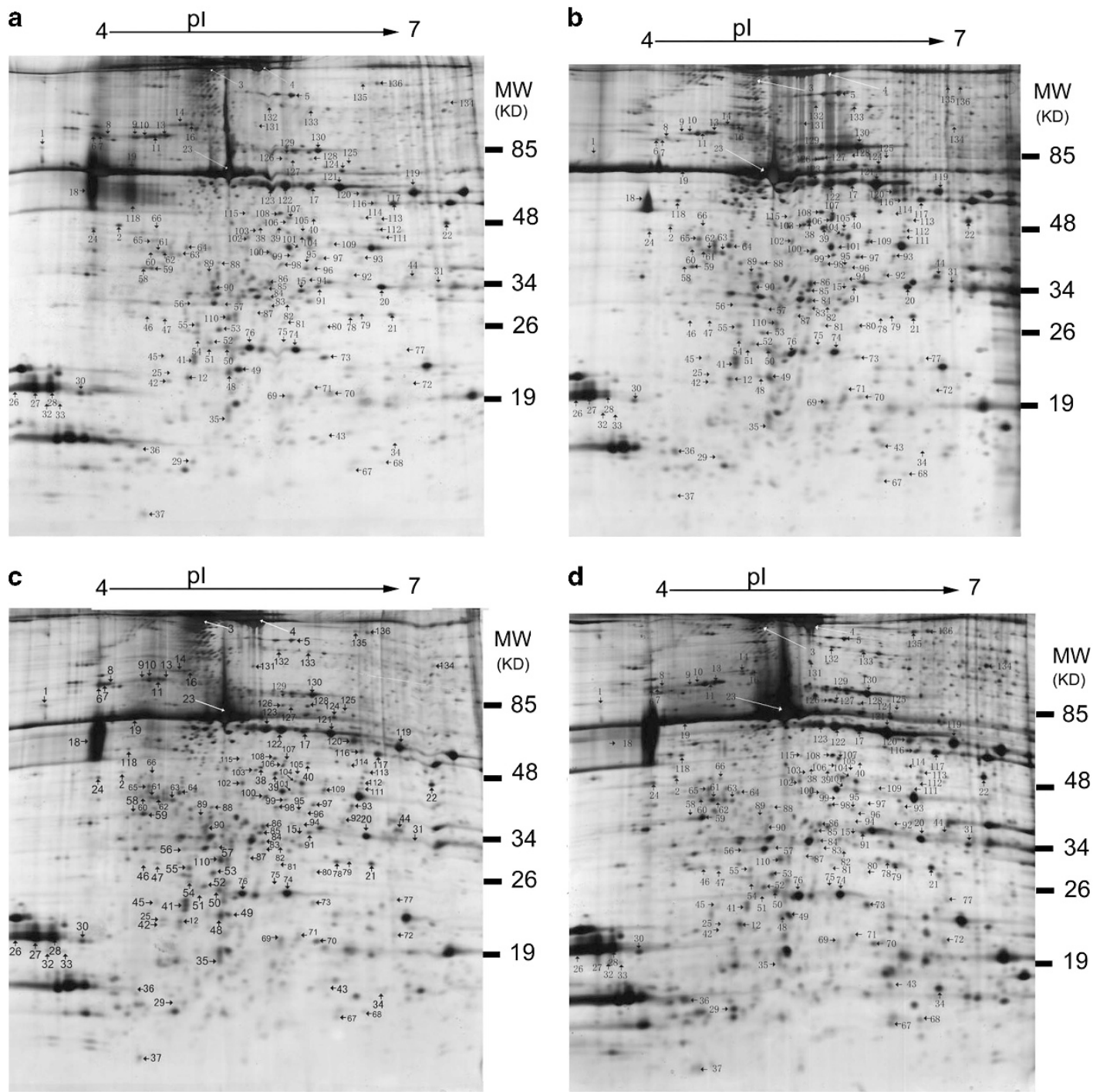

Figure 1 Representative image of 2-D silver-stained proteins (120 $\mu$ g each load) from abalone muscle and separated on a linear IPG strip (pH 4-7, 18 cm) in the first dimension and on a $12.5 \%$ sodium dodecyl sulfate-polyacrylamide gel in the second dimension. The molecular weights and pl scales are indicated. Each gel is representative of three independent replicates. Arrows indicate the positions of protein spots. Images of 2-DE from (a) $H$. discus hannai $q \times H$. discus hannai ơ (DD) muscle proteins, (b) $H$. gigantea $\$ \times H$. gigantea ơ (GG) muscle proteins and (c) $H$. discus hannai $q \times H$. gigantea ơ (DG) muscle proteins. (d) $\mathrm{H}$. gigantea $\$ \times H$. discus hannai ơ (GD) muscle proteins. Gel images selected from one of each three replicate samples. Spot numbering scheme depicts spots selected for MS identification, based on Table 3.

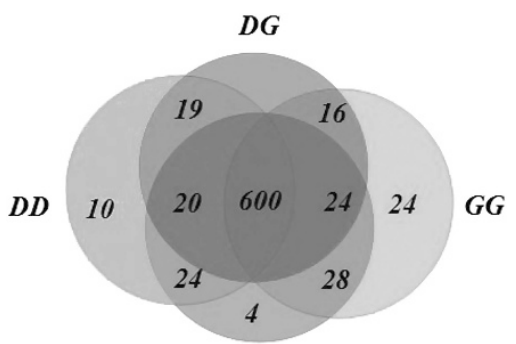

$G D$

Figure 2 Differentially expressed protein spots distributed among four abalone lines based on 2-DE gel image analysis. Different numbers of unique, paternally inherited, maternally inherited and relatively constant spots were characterized in Venn diagrams.
F1 hybrids and female abalone. Data show that maternal effects are conferred to abalone muscle proteins.

Analysis based on abalone muscle proteomic data in 2-DE spots revealed a primary mirror relationship (almost all stained spots in $\mathrm{F}_{1}$ hybrid muscle were found on 2-DEs from its parents) (Xie et al., 2006) among spots of purebreds and offspring (Figure 2). Comparisons indicated that almost all 2-DE spots from DG or GD depended on their purebreds.

\section{Analysis of data set of 'spot' and 'genotype'}

Variation in spot intensity in identically located spots due to small modifications can be treated as a normal phenotypic variation. Use of spots detected in all abalone also helps to avoid the expression of variation artifacts due to allelic variations that modifies protein charge 
(Diz and Skibinski, 2007). To compare the protein expression between abalone from hybrids and their parental lines, 110 sharp spots (non-ambiguous and detected in all the samples) were chosen from gels. Spots in crowded areas or around gel edges and spots with extremely high/low intensities were discarded. Protein spots unique for an individual were also discarded (qualitative changes). Spots used

Table 1 ANOVA analysis

\begin{tabular}{lrrrrr}
\hline Source of variation & Sum of squares & $d f$ & Mean square & $F$ & $P$ \\
\hline Genotype (P) & 12.610 & 3 & 4.203 & 5.683 & 0.001 \\
Spot (S) & 247.553 & 109 & 2.271 & 3.071 & $<0.001$ \\
Error & 650.897 & 880 & 0.740 & & \\
\hline
\end{tabular}

Abbreviation: ANOVA, analysis of vaiance.

Color Key

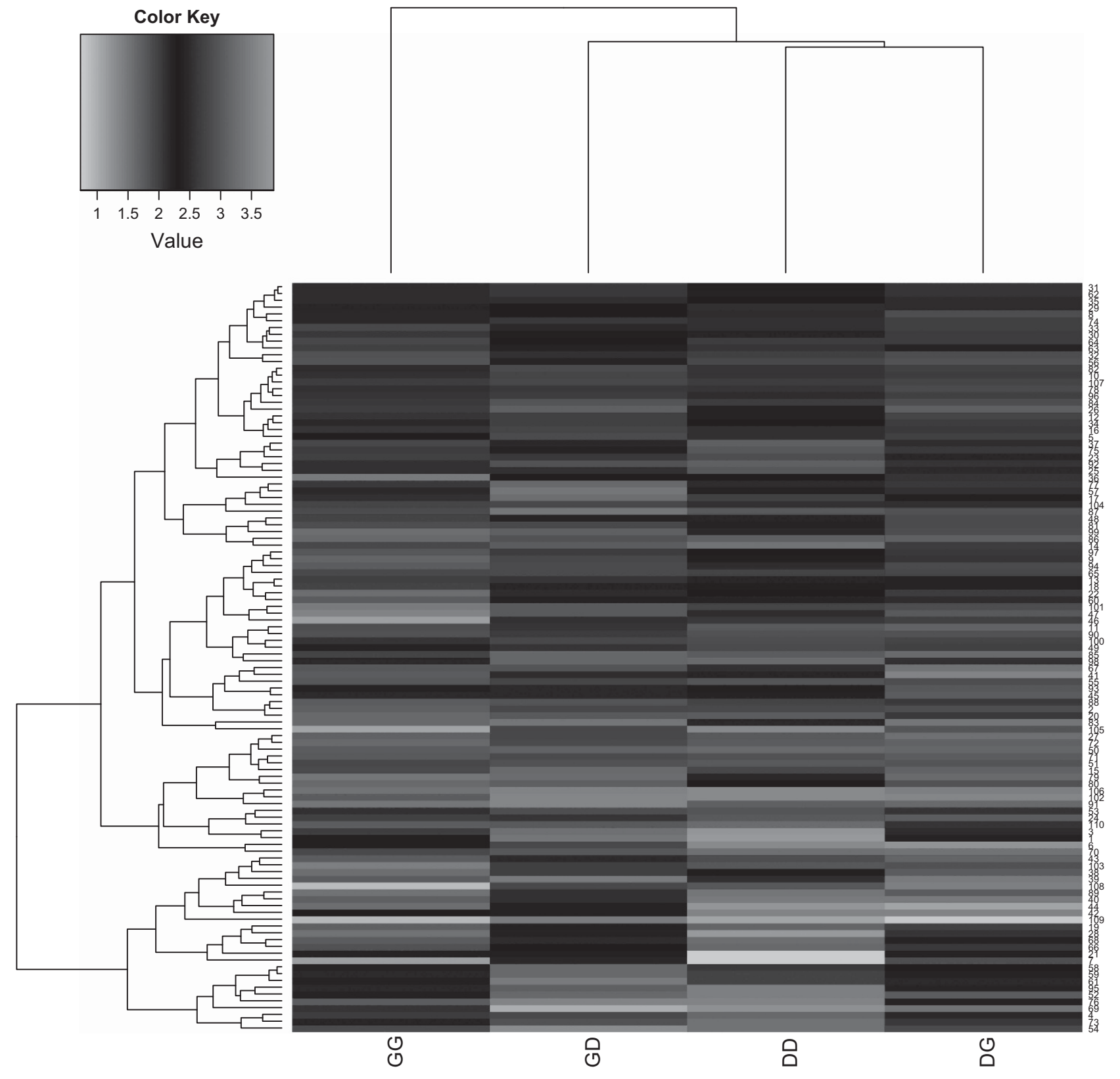

were similar to those used in previous experiments with mussels (Mosquera et al., 2003). A fully factorial analysis of variance (type III sums of squares) was carried out according to previous recommendations (Karp et al., 2005) using the 'spot' and 'genotype' factors, the latter being a generic term for the four types of abalone (DD, DG, GD and GG). Analysis of variance results are shown in Table 1. Both genotype and spots were highly significant $(P<0.01)$.

\section{Genetic distances of four abalone groups}

Hierarchical cluster analysis depicted patterns of similarities and differences between genotypes. The analysis was carried out with all 110 spots and normalized and standardized values. DD and DG were clustered together first and then clustered with GD, whereas the distance of DD and GG was maxima according to hierarchical cluster analysis (Figure 3).

Figure 3 Dendrogram of hierarchical cluster analysis on DD, GG, DG and GD. 
Table 2 Spot number from 2-D gel, number of spots and differential expression patterns between offspring and parents for 136 protein spot identity is presented as normalized percentage of spot volumes

\begin{tabular}{ll}
\hline Type & $\begin{array}{l}\text { Number Spot nos. } \\
\text { of spots }\end{array}$ \\
\hline
\end{tabular}

\begin{tabular}{lrl}
\hline$D D>D G>G D=G G$ & 1 & 57 \\
$D D>D G>G D>G G$ & 10 & $70,6,53,54,82,106,9,11,30,74$ \\
$D D>D G>G G>G D$ & 3 & $7,58,124$ \\
$D D>G D>D G>G G$ & 6 & $49,113,33,76,62,22$ \\
$D D>G D>G G>D G$ & 8 & $73,95,10,81,50,5,36,1$ \\
$D D>G G>D G>G D$ & 6 & $80,42,13,97,14,2$ \\
$D D>G G>G D>D G$ & 1 & 37 \\
$D G>D D>G D>G G$ & 2 & 85,110 \\
$D G>D D>G D>G G$ & 1 & 45 \\
$D G>D D>G G>G D$ & 5 & $48,55,87,90,122$ \\
$D G>G D>D D>G G$ & 5 & $136,125,128,35,59$ \\
$D G>G D>G G>D D$ & 2 & 60,21 \\
$D G>G D>G G>D D$ & 1 & 25 \\
$D G>G G>D D=G D$ & 1 & 15 \\
$D G>G G>D D>G D$ & 7 & $79,64,8,88,89,92,44$ \\
$G D>D D>D G>G G$ & 9 & $91,52,56,101,24,12,117,17,20$ \\
$G D>D D>G G>D G$ & 2 & 134,119 \\
$G D>D G>D D>G G$ & 12 & $131,127,129,28,26,27,114,126,78,46$, \\
& & 66,68 \\
$G D>D G>G G>D D$ & 12 & $72,116,77,65,102,93,32,67,47,51,34,130$ \\
$G D>G G>D D>D G$ & 3 & $133,135,132$ \\
$G D>G G>D G>D D$ & 4 & $43,108,118,121$ \\
$G G>D D>D G>G D$ & 5 & $71,96,99,109,38$ \\
$G G>D D>G D>D G$ & 4 & $84,86,115,31$ \\
$G G>D G>D D>G D$ & 9 & $63,94,41,16,105,69,61,103,40$ \\
$G G>D G>G D>D D$ & 7 & $83,100,111,112,98,123,39$ \\
$G G>G D>D D=D G$ & 1 & 29 \\
$G G>G D>D D>D G$ & 1 & 107 \\
$G G>G D>D G>D D$ & 3 & $75,104,120$ \\
Supersaturation & 5 & $4,19,23,3,18$ \\
\hline$T P I$ &
\end{tabular}

Type is the expression pattern of differentially expressed protein spots in hybrids compared with their parental lines. Spots number is the identifying number of the differentially expressed protein spots on each 2D map.

\section{Identification of 2-DE differentially expressed protein spots}

Three criteria were used to screen for proteins differentially expressed among the four types of abalone (DD, DG, GD and GG). First, spots in crowded gel areas, at gel borders or in areas with streaking were avoided. Second, spots only detected in one (or two or three) abalone populations were considered differentially expressed protein spots. Third, for the spots detected, only those whose percentage volume varied more than twofold among the four types of abalone were selected. Because very faint spots were avoided, perhaps those selected were dominated by housekeeping proteins that tend to have high expression (Gygi et al., 2000). In addition, some housekeeping proteins were selected, and 136 gel spots were identified from 2-DE gels of muscle samples from the four abalone groups. A total of 43 spots matched abalone proteins $(31.6 \%$ identification rate). Identification results from MS/MS are summarized in Supplementary Table S1.

The 136 gel spot expression patterns are summarized in Table 2. Spots identified in a proteomics study can be assigned ontology terms relating to protein identity, function and process. The identified proteins were then matched to specific processes or functions by searching Gene Ontology (GO) (www.geneontology.org).
Protein spot distributions fell into three general categories: additivity, over-dominance, and underdominance. Additivity refers to expression between the two parental lines in their offspring, overdominance is an expression higher than both parental lines, whereas the opposite is true for underdominance (Wang et al., 2008). According to Table 2, 27 spots form hybrids had additivity; and 32 hybrid spots had over-dominance, leaving 16 spots in DG with overdominance and 18 spots in GD with over-dominance. In these 136 identified gel spots, hybrid additivity or over-dominance accounted for $68.38 \%$.

\section{Identification of differentially expressed proteins}

Identified proteins were matched to specific processes or functions by searching a Gene Ontology database (www.geneontology.org). The 136 identified gel spots had many functional classifications (Figure 4). Most differentially expressed proteins were involved in ATP binding, protein binding, motor protein, muscle protein, myosin, motor activity, glycometabolism, muscle cell homeostasis and response to stress.

Spot numbers from 2-D gel, putative protein identification, functional classification and differential expression pattern between offspring and its parents for protein identity are presented with normalized average percentage of spot volumes (protein abundance, which is the sum of pixel intensity within the spot boundary) and $P$-values (Table 3). 2-D images were analyzed on the PDQuest software. Normalized spot volumes, that is, the volume of each spot over the volume of all spots in the gel, were used for comparison of the different groups.

Fourteen protein spots were in the category of energy metabolism (Table 3). Expression of spots was additive or over-dominant in GD, which includes almost every group for energy metabolism. Spots involved in energy metabolism were additive or over-dominant in the offspring.

For stress-responsive proteins, we observed over five different types (Table 3): 70-kD heat-shock protein and $\mathrm{Cu} / \mathrm{Zn}$-superoxide dismutase (SOD) were additive in the offspring, and macrophage migration inhibitory factor (MIF), Mn-SOD, and sigma class glutathione-Stransferase 2 were greatly improved in the reciprocal cross F1. Cathepsin L-like cysteine proteinase was lower in F1.

\section{Subcellular location of identified proteins}

Subcellular locations of 136 identified gel spots are summarized in Figure 5. Membrane proteins included prohibitin and 3D domain protein; endoplasmic reticulum protein included cytochrome b5; mitochondrion proteins included ATP synthase beta subunit, malate dehydrogenase precursor, Mn-SOD and glyceraldehyde 3-phosphate dehydrogenase; periplasm protein included peptidyl-prolyl cis-trans isomerase; extracellular protein included cathepsin L-like cysteine proteinase and other proteins that were mainly in the cytoplasm.

\section{DISCUSSION}

Cross-breeding is necessary for aquaculture (Zhang et al., 2007) as interaction and complementation of different dominant genes from parents can create better offspring. In our previous study, genetic changes of intraspecific hybridization between Haliotis diversicolor Reeve two populations were revealed (Di et al., 2013). Here we present data to suggest that a proteomic approach can predict abalone interspecific hybridization. Our data suggest that compared with intraspecific hybridization, the result may show more maternal effect in interspecific hybridization. 


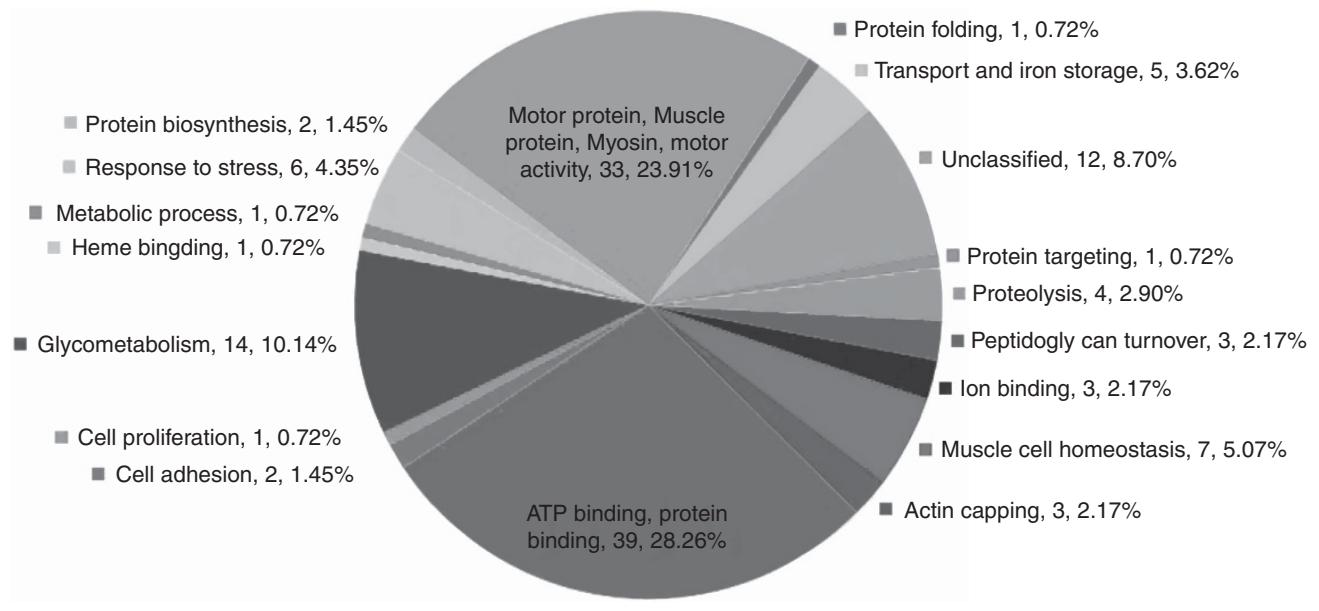

Figure 4 The GO functional categories of the protein spots identified by MS/MS in abalone muscle. Pie chart showing the distribution of the proteins identified between offspring and parents according to their functions. Protein groups were categorized based on putative functions.

Generally, phenotypic characterization is a precondition for abalone breeding. Intact proteins are fundamental phenotype units, and 2-DE gels can be used for such genetic investigations and genetic protein variability can be detected qualitatively and quantitatively (De Vienne et al., 2001). Spot intensities are proportional to relative protein concentrations in the cell, so those differences reflect natural genetic variability for protein expression. PD Quest software can be used to quantify spot intensities and, along with appropriate calibration methods and statistical tests, allow this variability to be assessed (De Vienne et al., 2001).

We primarily tested the hypothesis that protein in abalone muscle would vary among different abalone groups and that a gene transmitted from the parents to the filial generation would be mirrored among the four groups. Thus, if a gene signal was shared by both female parent and filial generation but not by the male parent, the genetic relationship would be easy to determine and vice versa. In Figure 2, in DG, 39 spots from DD do not exist in GG. Our 2-DE images strongly indicated that the different protein spots had a good mirror relationship from the parents to the filial generation; however, it required more data to be confirmed. There were four unique spots in GD that were relatively weak overall and spatially close to major shared spots. We suspected that these four unique spots might be regulated by posttranslational modifications of the nearby major spots with a pI shift and a molecular weight increase or due to size variation of active paralogs of the genes.

Of note, many proteins identified had multiple isoforms in the 2-DE gel with different $\mathrm{pI}$ and molecular weight values, forming a train of spots horizontally or scattering on the 2-DE gel. For example ATP synthase beta subunit was identified in the 2-DE gel (Table 3, spots 9, 10 and 11) with different pI values, and they had the same amino-acid sequence. So, multiple forms of a gene product existed in the proteome. Many isoforms are caused by single-nucleotide polymorphism or small genetic differences between alleles of the same gene. Currently, we cannot determine whether these isoforms are physiologically relevant, but the existence of multiple isoforms opens new areas for understanding gene functions. Proteins from 2-DE patterns can also be linked to their genes that allows one of the map genes encoding various characters of proteins (Klose et al., 2002). In addition to insufficient protein separation, the mature form of a protein depends not only on its gene but also on posttranslational modification. Therefore, a parental gene transmitted to the next generation can be directly translated into its own product, and it also can be indirectly converted into protein form through a series of modifications. Our data indicate that protein modification signals present genetic information useful for analysis. Statistics can be used to study the overall trend of expression levels of such proteins. In total, 136 gel spots were identified, and important proteins are described below.

\section{Energy metabolism proteins}

ATP synthase is needed for cellular energy interconversion and the $\beta$ subunit is the catalytic center. ATP synthase beta subunit (spot 9 and spot 11) expression patterns are $\mathrm{DD}>\mathrm{DG}>\mathrm{GD}>\mathrm{GG}$. In invertebrates, arginine kinase is a phosphagen kinase and a phosphotransferase with a role in energy metabolism. Expression of arginine kinase (spot 17) exhibited over-dominance in GD $(P<0.05)$, but there is no significant difference between DD, GG and DG. Triosephosphate isomerase (spot 20) is a glycolytic enzyme. In four abalone families, expression was: GD $>$ DD $>$ DG $>$ GG, and expression exhibited overdominance in $\mathrm{GD}(P<0.05)$ but with no significant differences among DD, DG and GG. A malate dehydrogenase precursor (spot 22) expression was no different in the four abalone families. Glyceraldehyde 3-phosphate dehydrogenase (spot 117) is a key enzyme in the glycolytic pathway. Expression exhibited over-dominance in GD $(P<0.05)$ but additivity in DG. Fructose-bisphosphate aldolase (spot $119)$ is needed in glycolysis and gluconeogenesis. Greater expression of fructose-bisphosphate aldolase was observed, and expression exhibited over-dominance in GD $(P<0.05)$ and underdominance in DG. Protein spot 130 was enolase, and expression exhibited overdominance in offspring.

We observed spots 17, 20,117, 119, 130 and 132 with the highest expression in GD, which cover almost every group of energy metabolism function. This may be related to fast growth of GD. Proteins involved in energy metabolism are additive or over-dominant in the offspring, suggesting that a positive mid-parent heterosis and over-parent heterosis for growth were improved in a reciprocal cross $F_{1}$. This pattern implied that dominant inheritance from DD to $F_{1}$ may be complemented in the offspring. 


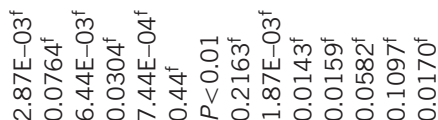

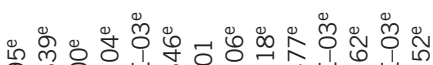

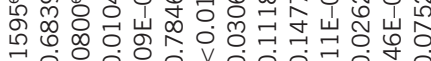

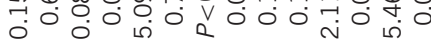

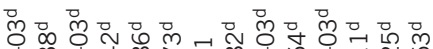

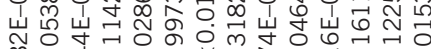

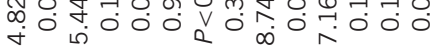

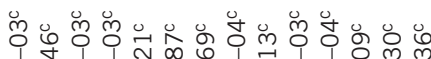

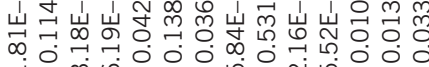

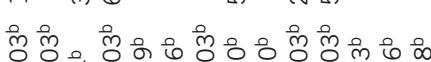
山山山 $\omega^{\infty} \infty$

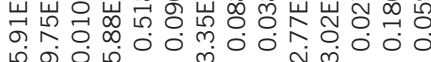

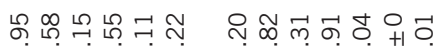

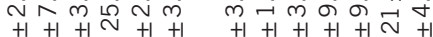

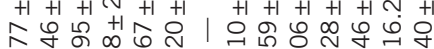

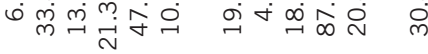

웅 @ -

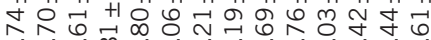

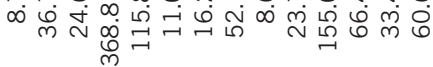

m®

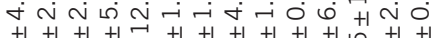
m 岗 ๓

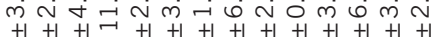

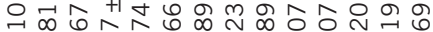

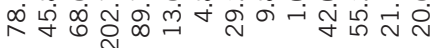

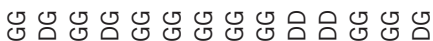

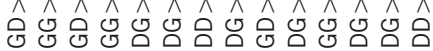

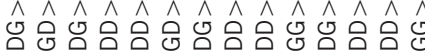

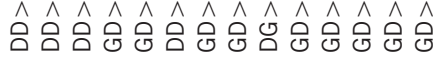

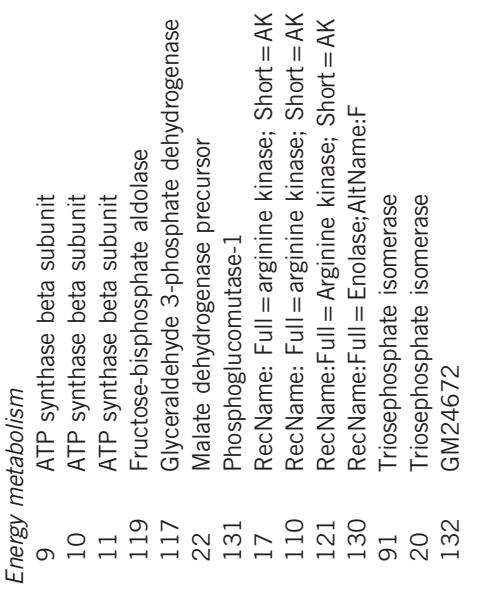

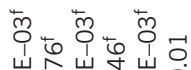

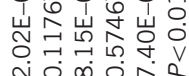

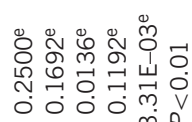

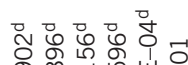

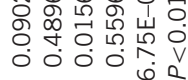

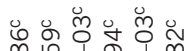

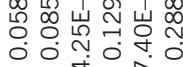

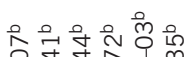

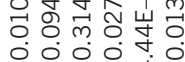

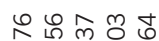

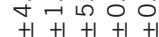
$\infty$ अं山्यूं०

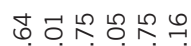
× i min mo

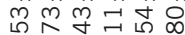

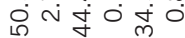

공요요웡 iom

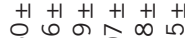
穴

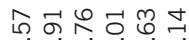

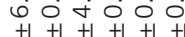

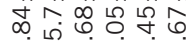
造

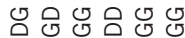

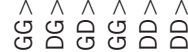

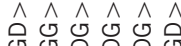

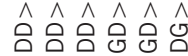

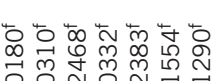

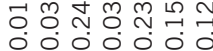
N

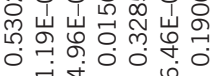

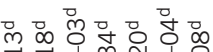

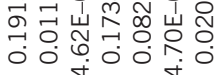

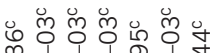
Ő

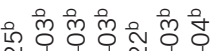

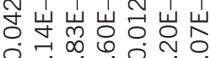
O

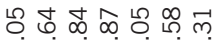

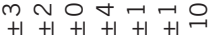

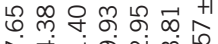

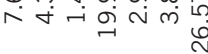

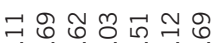

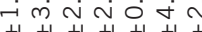

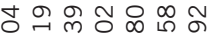

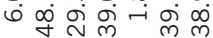

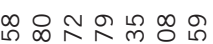

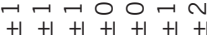

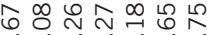
추웝

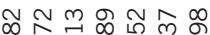

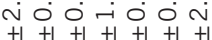

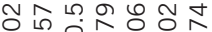
வ่

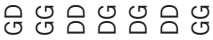

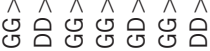
$\hat{\mathrm{O}} \hat{\mathrm{O}} \hat{\mathrm{O}} \hat{o} \hat{o} \hat{o} \hat{o}$

ôôôooㅇoㅇo용

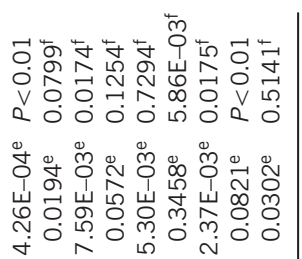

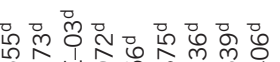

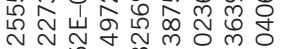

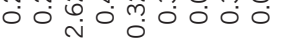

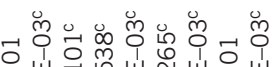

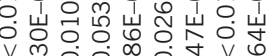

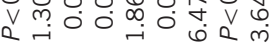

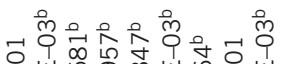

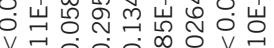
v 임

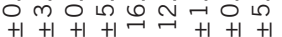

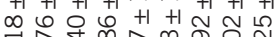
$0 \infty$ 守 $\underset{\sim}{\infty} \infty$

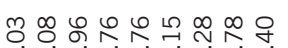
o $+\dot{+} \dot{m} m$ mi

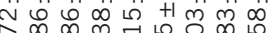

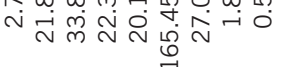

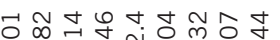
O언

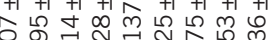

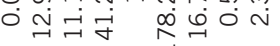

为 0 | ○ण

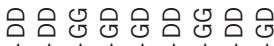

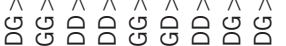
.

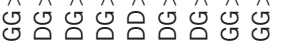

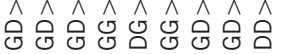

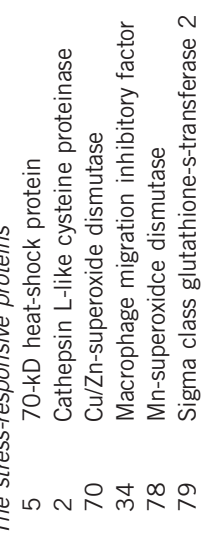

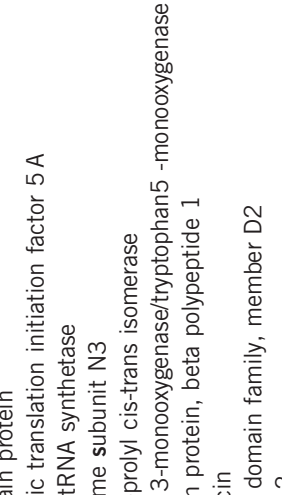

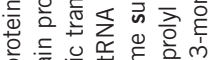

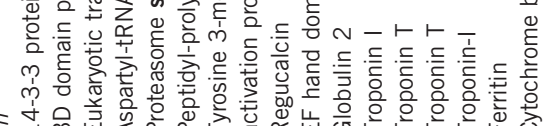

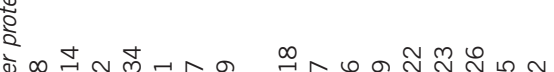

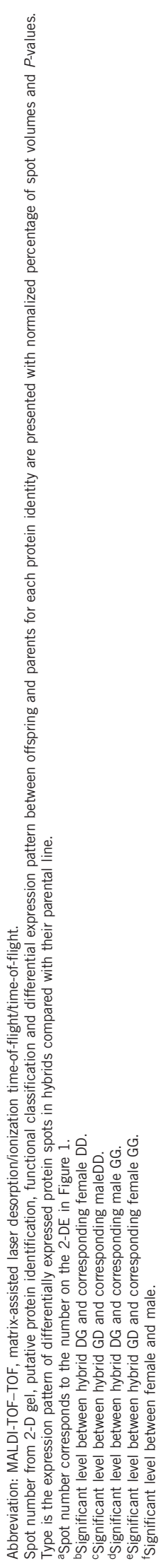




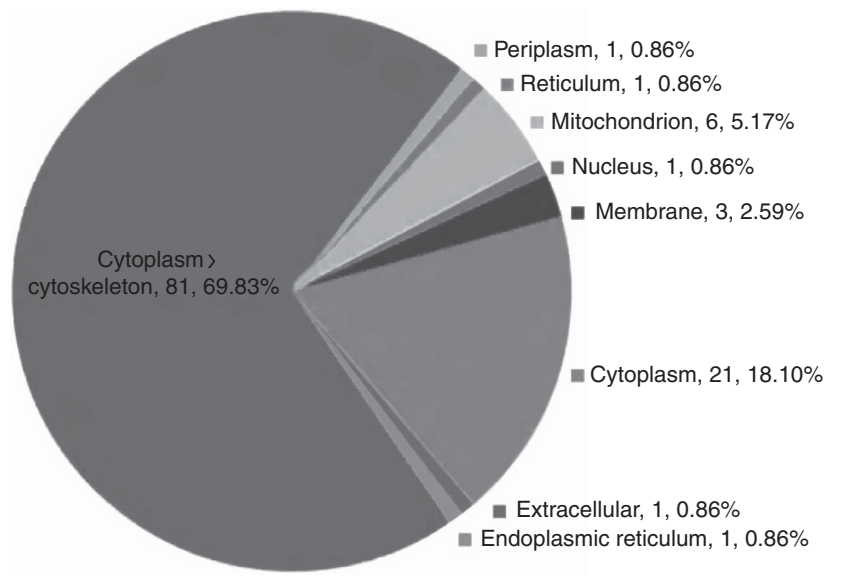

Figure 5 Pie chart showing the distribution of the proteins identified between offspring and parents according to their subcellular localization.

\section{Stress-responsive proteins}

Heat-shock protein 70 (Hsp70) (spot 5) is a family of highly conserved stress proteins that protects cells from harmful assaults. Expression of Hsp70 showed additivity in GD.

$\mathrm{Cu} / \mathrm{Zn}-\mathrm{SOD}$ (spot 70) is closely related to shellfish immunity and can enhance the ability of phagocytic cells and immune function and make cell form reactive oxygen species poisoning (Kim et al., 2007). Expression of $\mathrm{Cu} / \mathrm{Zn}-\mathrm{SOD}$ in the DD offspring show additivity. Mn-SOD (spot 78) is an important first-line antioxidant defense enzyme scavenging oxygen-free radicals and thus involved in cellular defense against oxidative stress. Expression of $\mathrm{Mn}-\mathrm{SOD}$ in the offspring show over-dominance. In short, SOD expression was additive and over-dominant in hybrid offspring. Li (2010) showed that SOD activity of DG hemolymph was over-parent heterosis, which was consistent with the study of SOD protein expression in the muscle. Data here suggest that hybrid offspring have mid-parent or over-parent heterosis.

Macrophage MIF (spot 34) is a multifunctional protein that can act as a cytokine, ahormone, a growth factor or an enzyme. It has a clear role as a particular cytokine and a mediator in acute and chronic inflammatory diseases, cell proliferation, autoimmune diseases and tumor angiogenesis (Lue et al., 2002). MIF is an old, conserved biomolecule, likely connected with immune evolution. This conservation of MIF may be caused by its functional importance (Du et al., 2004). MIF expression showed over-dominance in hybrid offspring.

Glutathione-S-transferase (spot 79) has detoxifying effects. Expression of spot 79 in GG may be very low, so this protein was not detected in the 2-DE. Hybrid offspring show over-dominance, and expression of glutathione-S-transferase in DG is about five times as high as that in female parent DD.

For stress-responsive proteins, spots 5 and 70 were additive in the offspring. Spots 34, 78 and 79 exhibited over-parent heterosis and were increased in reciprocal cross F1. Thus three of the six differentially expressed protein spots were upregulated in the offspring, two spots were additive and one spot was downregulated (Table 3). This may be related to the survival rate of the offspring. We demonstrated previously (Luo et al., 2012) that the survival rate of the reciprocal hybrid was significantly higher than that of $H$. discus hannai, suggesting that the hybrid had higher heterosis.

$H$. gigantea had a wide range of optimum temperatures and strong disease resistance, while $H$. discus hannai grows well but has weak high-temperature resistance (Gao et al., 2000). The two species are complementary with respect to some traits, so we expected to breed a new variety of abalone with high-temperature resistance, fast growth and strong disease resistance through hybridization. The proteins identified, in addition to structural proteins, had different expression and various physiological functions, mainly related to metabolism and stress-responsive proteins. For proteins related to energy metabolism (spots 9, 11, 20, 22 and 117), expression in the offspring was higher than in GG, and this may have been related to growth. Most stressinduced proteins exhibited over-dominance or additivity (spots 5, 34, 70, 78 and 79), and this may have been related to disease resistance. Genetic analysis of proteomes may reveal types of polymorphism that are most relevant in disease-association studies.

\section{Other proteins}

Protein spot 118 was identified as regucalcin, which is a calcium $\left(\mathrm{Ca}^{2+}\right)$-binding protein that is multifunctional in many organisms. $\mathrm{Ca}^{2+}$ is involved in many physiological processes of invertebrates and is the primary cation for shell structures. It is a second messenger for the regulation of muscle contraction, neuronal activation, cell differentiation and cell death. Many energy-dependent $\mathrm{Ca}^{2+}$ transporters and $\mathrm{Ca}^{2+}$ channels exist to mediate intracellular $\mathrm{Ca}^{2+}$ and $\mathrm{Ca}^{2+}$ signals (Osterloh et al., 1998). Of note, calmodulin, myosin light chain and troponin $\mathrm{C}$ can maintain intracellular $\mathrm{Ca}^{2+}$ homeostasis as well as transmit $\mathrm{Ca}^{2+}$ signals to regulate specific target proteins (Osterloh et al., 1998). Expression in our results was: GD $>$ GG $>$ DG $>$ DD. Expression of regucalcin is the greatest in GD. Protein spot 47 was identified as EF hand domain family protein. It is associated with calcium signaling, and its pattern of expression in the four groups was: $\mathrm{GD}>\mathrm{DG}>\mathrm{GG}>\mathrm{DD}$. Offspring show upregulation of this protein. $\mathrm{Li}$ (2010) showed that the $\mathrm{Ca}^{2+}$ in the two kinds of hybrid offspring foot muscle is slightly higher than in the parent, and $\mathrm{Ca}^{2+}$ contents were $104.43,149.76,155.03$ and $103.86\left(10 \mu \mathrm{gg}^{-1}\right)$ in DD, DG, GD, and GG muscle, respectively. That it is highest in GD muscle is consistent with the expression of regucalcin. Data show that the reciprocal cross offspring GD may have an advantage in muscle contraction and other functions. Luo (2009) studied shell shape features of the abalone $H$. gigantea and $H$. discus hannai. The shell of $H$. gigantea is thick, and the shell of $H$. discus hannai is thin. Here the expression of regucalcin in the GG muscle is higher than that in the DD muscle, which is consistent with shell thickness.

Protein spot 67 was identified as peptidyl-prolyl cis/trans isomerase. This novel postphosphorylation signaling regulator sits at the crossroad of many signaling pathways controlling cell proliferation and transformation (Lei et al., Peptidyl prolyl cis/trans isomerase has crucial roles in cell cycling, neural pathology and immune response. Its expression pattern in the four groups was: $G D>D G>G G>D D$, and the offspring exhibited upregulation.

Protein spot 72 was identified as eukaryotic translation initiation factor 5A (eIF5A). eIF5A is a small acidic protein that is highly conserved from archaea to mammals. eIF5A promotes the formation of the first peptide bond at the onset of protein synthesis (Singh et al., 2014). It is not only involved in translation initiation but is also involved in the occurrence of cancer in the human body, cell proliferation, senescence, cell death and environmental responses of plants. eIF5A expression pattern in the four groups was: $\mathrm{GD}>\mathrm{DG}>\mathrm{GG}>\mathrm{DD}$, and the expression was upregulated in the offspring.

Protein spot 25 was identified as ferritin. Ferritin is an iron-binding protein, which is important in iron metabolism and sequestration and therefore in the maintenance of homeostasis of iron levels in the body (Kim et al., 2012). However, recent reports demonstrate that ferritin is 
involved in several functions, such as cell activation, development, immunity and angiogenesis (Kim et al., 2012). Ferritins have been known to be involved in innate immunity through its characteristic iron-withholding ability (Kim et al., 2012). Several results supported a role for ferritin as a protectant against oxygen-free radical-mediated damage (Li et al., 2008). Ferritin expression in the four groups was $\mathrm{GD}>\mathrm{GG}>\mathrm{DG}>\mathrm{DD}$. Ferritin expression exhibited over-dominance in GD and additivity in DG.

Protein spot 58 was identified as 14-3-3 proteins, which have a role in cell signal transduction pathways through controlling interaction with targeting proteins. The 14-3-3 proteins are a family of conserved phospho-specific-binding proteins involved in diverse physiological processes. The 14-3-3 proteins form a family of highly conserved, acidic, dimeric proteins with a subunit mass of approximately $30 \mathrm{kDa}$ that are involved in protein interactions mediating signal transduction pathways. 14-3-3 has an important role in innate immunity in Drosophila (Shandala et al., 2011). 14-3-3 protein expression in the four groups was $D D>D G>G G>G D$, and the expression showed additivity in DG and underdominance in GD.

Protein spot 67 has crucial roles in cell cycling, spot 72 is involved in translation initiation, spot 25 is important in iron metabolism and sequestratingand spot 58 has a role in cell signal transduction pathways. Moreover, protein spots 67, 72, 25 and 58 are involved in the immune response. Expression of protein spots 67 and 72 exhibited upregulation in the offspring. Expression of spot 25 exhibited overdominance in GD and additivity in DG. We demonstrated previously that the growth rate of hybrids was faster than $H$. discus hannai and $H$. gigantean after 100 days. The survival rate of the reciprocal hybrid was significantly higher than $H$. discus hannai (Luo et al., 2012). These proteins may be related to the growth rate and survival rate of offspring.

Protein markers can help assess genetic variability and establish genetic distances and phylogenetic relationships between lines, species and genus (Thiellement et al., 1999). Here we identified 136 differentially expressed protein spots and focused on discussion of proteins involved in energy metabolism and stress response. Most energy metabolism proteins were additive, and stress-induced proteins displayed additivity or over-dominance. Future studies will establish an evaluation system of heterosis using such 'protein markers'.

\section{CONCLUSION}

In conclusion, protein expression was observed in muscle samples using 2-DE and MALDI-TOF-TOF analyses. Different 2-DE image muscle protein spots had a mirrored relationship between purebreds and the $F_{1}$ hybrid. Most energy metabolism proteins were additive, and stress-induced proteins displayed additivity or over-dominance. These data will lay the foundation for future studies with proteomic methods to explore the molecular mystery connected with the heterosis of hybrid abalone.

\section{DATA ARCHIVING}

There were no data to deposit.

\section{CONFLICT OF INTEREST}

The authors declare no conflict of interest.

\section{ACKNOWLEDGEMENTS}

This work was supported by grants from the Hi-Tech Research and Development (863) Program of China (No. 2012AA10A412), NSFC (No. 31101896, 41106120), NSFC (No. U1205121). the Earmarked Fund for Modern Agro-industry Technology Research System (No. CARS-48), Henan
Science and Technology Program (No. 14B240003) and the PhD Start-up Fund of Henan Normal University (No.qd13053). We are grateful to Professor John Hodgkiss for his help with English.

Dahal D, Mooney BP, Newton KJ (2012). Specific changes in total and mitochondrial proteomes are associated with higher levels of heterosis in maize hybrids. Plant $\mathrm{J}$ 72: $70-83$.

De Vienne D, Bost B, Fiévet J, Zivy M, Dillmann C (2001). Genetic variability of proteome expression and metabolic control. Plant Physiol Biochem 39: 271-283.

Di G, You W, Yu J, Wang D, Ke C (2013). Genetic changes in muscle protein following hybridization between Haliotis diversicolor reeve Japan and Taiwan populations revealed using a proteomic approach. Proteomics 13: 845-859.

Diz AP, Skibinski DO (2007). Evolution of 2-DE protein patterns in a mussel hybrid zone. Proteomics 7: 2111-2120.

Du JC, Xie XJ, Chen HP, Yang WL, Dong ML, Su J et al. (2004). Macrophage migration inhibitory factor (MIF) in Chinese amphioxus as a molecular marker of immune evolution during the transition of invertebrate/vertebrate. Dev Comp Immunol 28: 961-971.

Eldakak M, Milad SI, Nawar AI, Rohila JS (2013). Proteomics: a biotechnology tool for crop improvement. Front Plant Sci 4: 1-12.

Elliott NG (2000). Genetic improvement programmes in abalone: what is the future? Aquacult Res 31: 51-59.

Fu Z, Jin X, Ding D, Li Y, Fu Z, Tang J (2011). Proteomic analysis of heterosis during maize seed germination. Proteomics 11: 1462-1472.

Gao SS, Wang Q, Wang RB, Li X (2000). Abalone. Liaoning Science and Technology Press: Shenyang, China, $14 \mathrm{pp}$.

Guo B, Chen Y, Zhang G, Xing J, Hu Z, Feng W et al. (2013). Comparative proteomic analysis of embryos between a maize hybrid and its parental lines during early stages of seed germination. PLOS ONE 8: e65867.

Gygi SP, Corthals GL, Zhang Y, Rochon Y, Aebersold R (2000). Evaluation of twodimensional gel electrophoresis-based proteome analysis technology. Proc Natl Acad Sci USA 97: 9390-9395.

Hoecker N, Lamkemeyer T, Sarholz B, Paschold A, Fladerer C, Madlung J et al. (2008). Analysis of nonadditive protein accumulation in young primary roots of a maize (Zea mays L.) F1-hybrid1 compared to its parental inbred lines. Proteomics 8: 3882-3894.

Jin X, Fu Z, Ding D, Li W, Liu Z, Hu Y et al. (2014). Proteomic analysis of plumules and coleoptiles in maize between hybrids and their corresponding inbred lines. Acta Physiol Plant 36: 355-370.

Karp NA, Spencer M, Lindsay H, O'Dell K, Lilley KS (2005). Impact of replicate types on proteomic expression analysis. J Proteome Res 4: 1867-1871.

Karr TL (2007). Application of proteomics to ecology and population biology. Heredity 100 : 200-206.

Kim KY, Lee SY, Cho YS, Bang IC, Kim KH, Kim DS et al. (2007). Molecular characterization and mRNA expression during metal exposure and thermal stress of copper/zinc- and manganese-superoxide dismutases in disk abalone, Haliotis discus discus. Fish Shellfish Immunol 23: 1043-1059.

Kim H, Sandaruwan Elvitigala DA, Lee Y, Lee S, Whang I, Lee J (2012). Ferritin H-like subunit from Manila clam (Ruditapes philippinarum): molecular insights as a potent player in host antibacterial defense. Fish Shellfish Immunol 33: 926-936.

Klose J, Nock C, Herrmann M, Stühler K, Marcus K, Blüggel M et al. (2002). Genetic analysis of the mouse brain proteome. Nat Genet 30: 385-393.

Li M, Saren G, Zhang S (2008). Identification and expression of a ferritin homolog in amphioxus Branchiostoma belcheri: evidence for its dual role in immune response and iron metabolism. Comp Biochem Physiol B Biochem Mol Biol 150: 263-270.

Li YL (2010). Studies on resistance related physiological and biochemical characteristics of interspecies hybrids between Haliotis discus hannai and $H$. sieboldii. Master's thesis, Xiamen University, Xiamen, China.

Luo X (2009). Study on genetic basis of hybridization between Haliotis sieboldii Reeve and Haliotis discus hannai Ino. PhD diss., Xiamen University, Xiamen, China. 189 pp.

Luo X, Ke CH, You WW, Wang DX, Chen F (2010). Molecular identification of interspecific hybrids between Haliotis discus hannai Ino and Haliotis gigantea Gmelin using amplified fragment-length polymorphism and microsatellite markers. Aquacult Res 41: 1827-1834.

Luo X, Ke C, You W (2012). Heterosis analysis on interspcific hybrids between Haliotis discus hannai and H. Gigantea. J Shellfish Res 31: 316-316.

Luo X, Ke C, You W (2013). Estimates of correlations for shell morphological traits on body weight of interspecific hybrid abalone (Haliotis discus hannai and Haliotis gigantea). J Shellfish Res 32: 115-118.

Lue HQ, Kleemann R, Calandra T, Roger T, Bernhagen J (2002). Macrophage migration inhibitory factor (MIF): mechanisms of action and role in disease. Microbes Infect 4: 449-460.

Mosquera E, Lopez JL, Alvarez G (2003). Genetic variability of the marine mussel Mytilus galloprovincialis assessed using two-dimensional electrophoresis. Heredity 90: $432-442$.

Muthreich N, Schützenmeister A, Schütz W, Madlung J, Krugc K, Nordheim A et al. (2010). Regulation of the maize (Zea mays L.) embryo proteome by RTCS which controls seminal root initiation. Eur J Cell Biol 89: 242-249.

Osterloh D, Ivanenkov VV, Gerke V (1998). Hydrophobic residues in the C-terminal region of S100A1 are essential for target protein binding but not for dimerization. Cell Calcium 24: $137-151$. 
Saeed AI, Bhagabati NK, Braisted JC, Liang W, Sharov V, Howe EA et al. (2006). TM4 microarray software suite. Methods Enzymol 411: 134-193.

Shandala T, Woodcock JM, Ng Y, Biggs L, Skoulakis EM, Brooks DA et al. (2011). Drosophila $14-3-3 \varepsilon$ has a crucial role in anti-microbial peptide secretion and innate immunity. J Cell Sci 124: 2165-2174.

Singh S, Raju K, Jatekar D, Dinesh N, Paul MS, Sobhia ME (2014). Leishmania donovani eukaryotic initiation factor 5A: molecular characterization, localization and homology modelling studies. Microb Pathog 73: 37-46.

Thiellement H, Bahrman N, Damerval C, Plomion C, Rossignol M, Santoni V et al. (1999). Proteomics for genetic and physiological studies in plants. Electrophoresis 20: 2013-2026.

Thiemann A, Meyer S, Scholten S (2009). Heterosis in plants: manifestation in early seed development and prediction approaches to assist hybrid breeding. Chin Sci Bull 54: 2363-2375.

Vasemägi A, Primmer CR (2005). Challenges for identifying functionally important genetic variation: the promise of combining complementary research strategies. $\mathrm{Mol}$ Ecol 14 3623-3642.
Wang W, Meng B, Ge X, Song S, Yang Y, Yu X et al. (2008). Proteomic profiling of rice embryos from a hybrid rice cultivar and its parental lines. Proteomics 8 $4808-4821$.

Wang DZ, Li C, Zhang Y, Wang YY, He Z P, Lin L et al. (2012). Quantitative proteomic analysis of differentially expressed proteins in the toxicity-lost mutant of Alexandrium catenella (Dinophyceae) in the exponential phase. J Proteomics 75: 5564-5577.

Xie Z, Wang J, Cao M, Zhao C, Zhao K, Shao J et al. (2006). Pedigree analysis of an elite rice hybrid using proteomic approach. Proteomics 6: 474-486.

Xiang X, Ning S, Wei D (2013). Proteomic profiling of rice roots from a super-hybrid rice cultivar and its parental lines. Plant Omics 6: 318-324.

Zhang H, Liu X, Zhang G, Wang C (2007). Growth and survival of reciprocal crosses between two bay scallops, Argopecten irradians concentricus Say and Argopecten irradians irradians Lamarck (1819). Aquaculture 272: S88-S93.

Zhang G, Que HY, Liu X, Xu HS (2004). Abalone mariculture in China. J Shellfish Res 23: 947-950

Supplementary Information accompanies this paper on Heredity website (http://www.nature.com/hdy) 